Signal \& Image Processing : An International Journal (SIPIJ) Vol.4, No.2, April 2013

\title{
COMPARATIVE ANALYSIS OF SKIN COLOR BASED MODELS FOR FACE DETECTION
}

\author{
Ekta Rewar ${ }^{1}$ and Saroj Kumar Lenka ${ }^{2}$ \\ ${ }^{1}$ Electronics and communication Engineering Department, MITS University, \\ Lakshmangarh, Sikar \\ ${ }^{1}$ ektarewar@yahoo.com \\ ${ }^{2}$ Information Technology Department, MITS University, Lakshmangarh, Sikar \\ ${ }^{2}$ lenka.sarojkumaregmail.com
}

\begin{abstract}
Human face detection plays an important role in many applications such as face recognition, human computer interface, biometrics, area of energy conservation, video surveillance and face image database management. The selection of accurate color model is the first need of the face detection. In this paper, a study on the various color models for face detection i.e. RGB, YCbCr, HSV and CIELAB are included. This paper compares different color models based on the detection rate of skin regions. The results shows that $\mathrm{YCbCr}$ as compared to other color models yields the best output even in varying lightening conditions.
\end{abstract}

\section{KEYWORDS}

Skin color model \& face detection.

\section{INTRODUCTION}

Detecting human faces automatically is becoming a very important task in many applications, such as content-based indexing video retrieval systems or security access control systems like the distributed audio visual archives network system [1]. Face detection is the first step in any automated system that solves problems such as facial expression recognition, face tracking and face recognition [2]. Up to now much work has been done on detecting and locating faces in color images and the methods like neural network based [3], level set methodology [4], chrominance based [5], ada boost based [6], skin color based [7] studied by many researchers. Among many face detection algorithms skin color model has been widely used because of simple performance, convenient use and high detection rate.

\subsection{Face Detection}

Face detection is a visual task which can be done by humans without any effort but in computer vision this task is very difficult. Given a single image, detect and localize the number of faces regardless of pose, illumination and expression. Face detection is used for self-serviced immigration clearance, for person verification, security, image search and research is being done in the area of energy conservation etc. The goal of face detection is to identify and locate all of the human faces regardless of their positions, scales, orientations, poses and light conditions and

DOI : 10.5121/sipij.2013.4206 
Signal \& Image Processing : An International Journal (SIPIJ) Vol.4, No.2, April 2013

this is a challenging problem because human faces are highly non-rigid with a high degree of variability of in size, shape, color and texture. A simple face detection block diagram is shown in fig.1:-

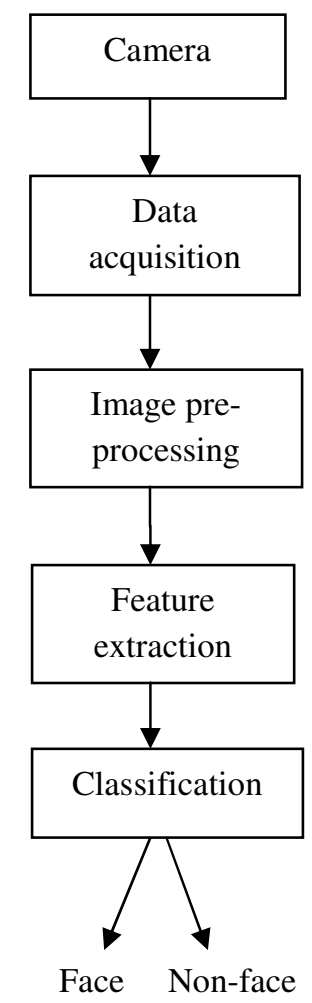

Figure 1. Basic block diagram of face detection

\subsection{Challenges in Face Detection}

Detection and recognition of faces are challenging because face has a wide variability in poses, shapes, sizes and texture. The problems or challenges in face detection are listed as follows [8]:-

$\begin{array}{ll}\text { - } & \text { Illumination } \\ \text { - } & \text { Facial Pose } \\ \text { - } & \text { Facial Expression } \\ \text { - } & \text { Motion } \\ \text { - } & \text { Occlusion } \\ \text { - } & \text { Image orientation } \\ \text { - } & \text { Image condition } \\ \text { - } & \text { Background Complexity } \\ \text { - } & \text { Environment Changes } \\ \text { - } & \text { Teise } \\ & \end{array}$


Signal \& Image Processing : An International Journal (SIPIJ) Vol.4, No.2, April 2013

These are the most general problems that occur during face detection. We can remove most of the drawbacks by increasing the efficiency and by applying the loops to get better results.

\section{SKIN COLOR BASED ALgORITHM}

Color is a useful piece of information for skin detection. It is the first most common approach for detecting meaningful skin color [9], as it avoids many exhaustive searches for faces in an entire image. Human skin colours are distinguished from different ethnic through the intensity of the skin colour not the chromatic features [10].According to the Hyun-Chul, Ju-Yeon You and SungII Chien, skin color algorithm can be used under the various illumination conditions [11] and according to Yogesh Tayal, Ruchika Lamba and Subhransu Padhee, color image is segmented into skin and non-skin region for different color spaces and the skin color algorithm can be applied on a wide variety of the images under different lighting conditions and with different backgrounds [12]. Many researches have been done using the color models. In this paper four basic color models i.e. RGB, HSV, YCbCr and CIELAB are reviewed.

\subsection{RGB Color Model}

RGB Colors are specified in terms of three primary colors i.e. Red (R), Green (G), and Blue (B). In RGB color space, a normalized color histogram is used to detect the pixels of skin color of an image and can be further normalized for changes in intensity on dividing by luminance. This localizes and detects the face [10].

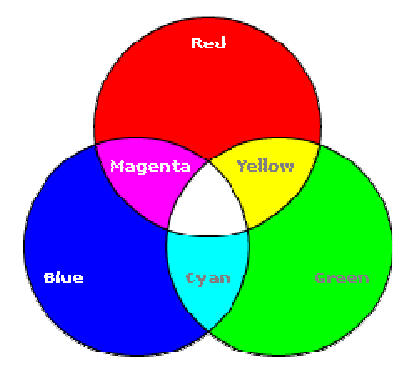

Figure 2. RGB color model

It is the basic color model and all other color models are derived from it. RGB color model is light sensitive. In comparison with other color models such as $\mathrm{YCbCr}$ or HSI it has a major drawback that it cannot separate clearly the mere color (Chroma) and the intensity of a pixel, so it is difficult to distinguish skin colored regions [2]. These factors contribute to the less favorable of RGB. It is widely used color space for processing and storing digital images because of the fact that chrominance and luminance components are mixed in RGB; it is not widely used in Skin detection algorithms [13]. 
Signal \& Image Processing : An International Journal (SIPIJ) Vol.4, No.2, April 2013

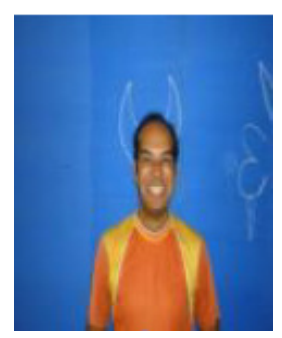

Input image

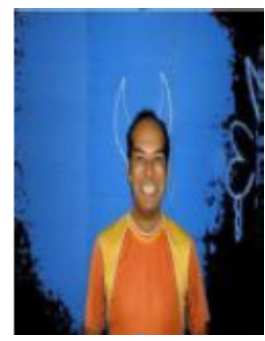

RGB image

Figure 3. RGB image

\subsection{HSV Color Model}

HSV Colors are specified in terms of Hue (H), Saturation (S) and Intensity value (V) which are the three attributes. Those are perceived about color. Hue refers to the color of red, blue and yellow having the range of 0 to 360 . Saturation means purity of the color and takes the value from 0 to $100 \%$ whereas Value refers to the brightness of color and provides the achromatic idea of the color [14]. From this color space, $\mathrm{H}$ and $\mathrm{S}$ will provide the necessary information about the skin color. The skin color pixel should satisfy the following condition:-

$$
0<=\mathrm{H}<=0.25
$$

$0.15<=\mathrm{S}<=0.9$

The transformation between HSV and RGB is non-linear [12]. In HSV color model Hue $(\mathrm{H})$ is not reliable for the discrimination task when the saturation is low. But where color description is consider, HSV color model is preferred over RGB model according to Yogesh Tayal [13], colors similarly is described that how human eye tends to perceive color and also describe color using more familiar comparisons such as vibrancy, color and brightness.

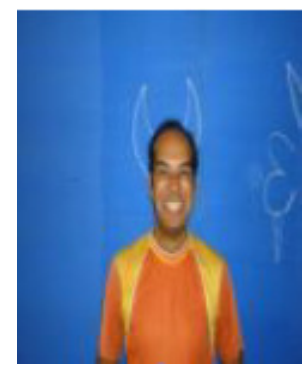

Input image

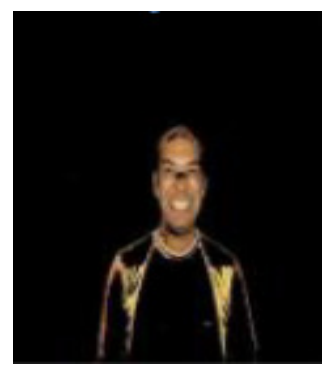

HSV image

\subsection{YCbCr Color Model}

Figure 4. HSV image

$\mathrm{YCbCr}$ Color model is specified in terms of luminance( $\mathrm{Y}$ channel) and chrominance $(\mathrm{Cb}$ and $\mathrm{Cr}$ channels). It segments the image into a luminous component and chrominance components [12]. In YCbCr color model, the distribution of the skin areas is consistent across different races in the $\mathrm{Cb}$ and $\mathrm{Cr}$ color spaces [15]. As RGB color model is light sensitive so to improve the performance of skin color clustering, $\mathrm{YCbCr}$ color model is used. Its chrominance components are almost independent of luminance and there is non-linear relationship between chrominance 
Signal \& Image Processing : An International Journal (SIPIJ) Vol.4, No.2, April 2013

$(\mathrm{Cb}, \mathrm{Cr})$ and luminance( $\mathrm{Y})$ of the skin color in the high and low luminance region [2]. Range of $\mathrm{Y}$ lies between 16-235 where 16 for black and 235 for white whereas $\mathrm{Cb} \& \mathrm{Cr}$ are scaled in the range of 16-240 [14]. The main advantage of YCCr color model is that influence of luminosity can be removed during processing of an image. Different plots for $\mathrm{Y}, \mathrm{Cb}$ and $\mathrm{Cr}$ values for face and non-face pixels were plotted using the reference images and studied to find the range of $\mathrm{Y}$, $\mathrm{Cb}$ and $\mathrm{Cr}$ values for the face pixels.

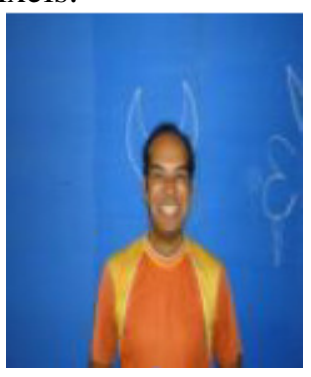

Input image

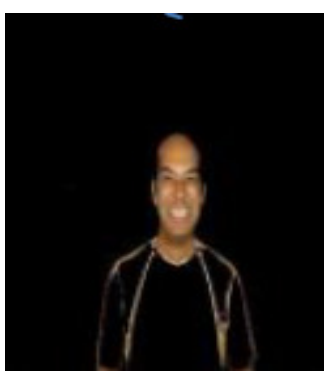

YCbCr image

Figure 5. YCbCr image

\subsection{CIELAB Color Model}

In 1976, the CIE (international commission on illumination) recommended the CIEL*a*b* or CIELAB, color scale for use. It provides a standard, approximately uniform color scale which could be used by everyone so that the color values can be easily compared. This color model is designed to approximate perceptually uniform Color spaces (UCSs). It is related to the RGB color space through a highly nonlinear transformation. Examples of similar color spaces are CIE-Luv and Farnsworth UCS [12]. It has three axes in it two are color axes and the third is lightness.

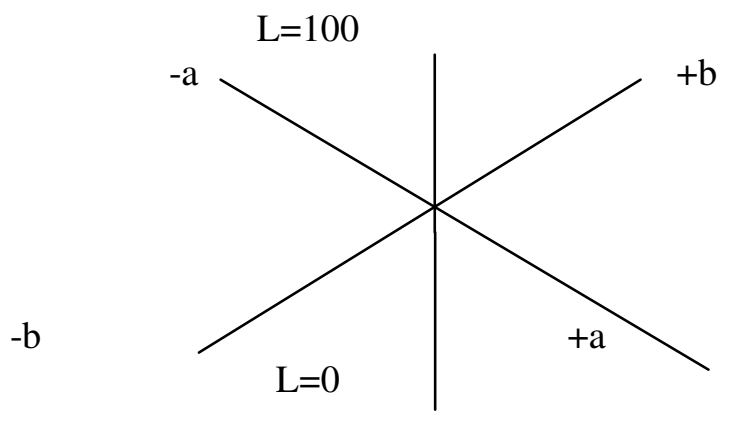

Figure 6. CIELAB Color Model

Where $\mathrm{L}$ indicates lightness, $+\mathrm{a}$ and $-\mathrm{a}$ indicates amount of green and red color respectively, $+\mathrm{b}$ and $-\mathrm{b}$ indicates amount of yellow and blue color respectively.

Here maximum value of $\mathrm{L}$ is 100 which represent a perfect reflecting diffuser (white color) and the minimum value for $L$ is 0 which represents black color. Axes a and $b$ do not have any specific numerical value. 
Signal \& Image Processing : An International Journal (SIPIJ) Vol.4, No.2, April 2013

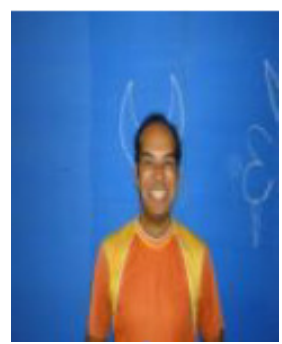

Input image

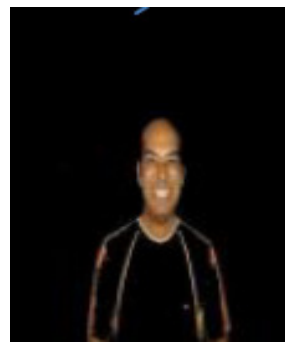

CIEL*a*b image

Figure 7. CIELAB image

\section{RESULTS AND COMPARISON}

The results after survey on these four color models are shown below in tabular format:-

Table 1. Comparison of four Color Models

\begin{tabular}{|l|l|l|}
\hline Type of color model & Detection rate \% & False detection \% \\
\hline RGB & 65 & 35 \\
\hline HSV & 70 & 30 \\
\hline CIELAB & 89 & 11 \\
\hline YCbCr & 90 & 10 \\
\hline
\end{tabular}

The table compares face detection rate of the four color models i.e. RGB, HSV, CIELAB and YCbCr. Detection rate of RGB color model is only $65 \%$ that means it can only detect $65 \%$ of the faces correctly. Detection rate of HSV color model is 70\%, CIELAB color model is $89 \%$ and detection rate of $\mathrm{YCbCr}$ color model is $90 \%$. Out of these four color model $\mathrm{YCbCr}$ has highest detection rate and lowest false detection rate.

To increase the speed, accuracy of the system and to overcome on false face detection rate these four color models can be combined to get better result, after combining all these models we can get correct detection rate which can be up to $92 \%$.

\section{CONCLUSION}

Four different color models in face detection have been studied and compared namely, RGB, $\mathrm{YCbCr}, \mathrm{HSV}$ and CIELAB. The paper concludes that out of these four color models $\mathrm{YCbCr}$ yields a better results and performance under varying lightening conditions and changes in illumination.

\section{REFERENCES}

[1] Rein-lien Hsu, Mohamed Abdel-Mottaleb \& Anil k. Jain, (2008) "Face Detection in Color Images", IEEE Transactions on Pattern Analysis and Machine Intelligence, Vol.24, No.5, pp696-706.

[2] Chandrappa D N, M Ravishankar \& D R Ramesh Babu, (2011) "Face Detection in Color Images using Skin Color Model Algorithm Based on Skin Color Information", IEEE Transactions, pp254258. 
Signal \& Image Processing : An International Journal (SIPIJ) Vol.4, No.2, April 2013

[3] Henry A. Rowley, Shumeet Baluja, \& Takeo Kanade, (1988) "Neural Neural Network-Based Face Detection", IEEE, pp1-28.

[4] M.Kumaravel, S.Karthik, P.Sivraj \& K.P.Soman, (2012) "Human Face Image Segmentation using Level Set Methodology", International Journal of Computer Applications, Vol. 44, No.12.

[5] Hongxun Yao \& Wen Gao, (2000) "Face detection and Location based on Skin Chrominance and Lip Chrominance Transformation from Color Images", Journal of Pattern Recognition Society, pp15551564.

[6] Jing-Ming Guo, Chen-Chi Lin, Min-Feng Wu, Che-Hao Chang \& Hua Lee, (2011) "Complexity Reduced Face Detection Using Probability-Based Face Mask Prefiltering and Pixel-Based Hierarchical-Feature Adaboosting", IEEE Signal Processing Letters, Vol.18,No.8, pp447-450.

[7] Jiang Oiang-rong \& Li Hua-Ian, (2010) "Robust Human Face Detection in Complicated Color Images", IEEE Transactions.

[8] Yang, M.H., Kriegman, D.J. \& Ahuja, "Detecting Faces in Images: A Survey", IEEE Transactions on Pattern Analysis and Machine Intelligence, Vol.24, No.1, pp34-58.

[9] Devendra Singh Raghuvansi \& Dheeraj Agrawal, (2012) "Human Face Detection by Using Skin Color Segmentation, Face Features and Region Properties", International Journal of Computer Application, Vol. 38, No.9, pp14-17.

[10] Nurulhuda Ismail, Mas Idayu \& Sabri, (2009) "Review of Existing Algorithms for Face Detection and Recognition", Proceedings of the 8th WSEAS International, pp.30-39.

[11] Hyun-Chul Do, Ju-Yeon You \& Sung-II Chien , (2007) "Skin Color Detection through Estimation and Conversion of Illuminant Color under Various Illumination", IEEE Transactions on Consumer Electronics, Vol.53, No.3, pp1103-1108.

[12] SonLam Phung, Abdesselam Bouzerdoum \& Douglas Chai, (2005) "Skin Segmentation using Color Pixel Classification: Analysis and Comparison", IEEE Transaction on Pattern Analysis and Machine Intelligence, Vol.27, No.1.

[13] Vivek Desai, Pranav Vankar, Jugal Mehta \& Ghanshyam Prajapati, (2012) "Face Detection Using Skin Color", International Conference on Computing and Control Engineering, ISBN No. 978-14675-2248-9.

[14] S. Chitra \& G. Balakrishnan, (2012) "Comparative Study for Two Color Spaces HSCbCr and YCbCr in Skin Color Detection", Applied Mathematical Sciences, Vol. 6, No. 85, pp4229 - 4238.

[15] Yogesh Tayal, Ruchika Lamba \& Subhransu Padhee, (2012) "Automatic Face Detection using Color Based Segmentation”, International Journal of Scientific and Research Publications, Vol.2, No.6, pp17.

\section{About Author (s):}

Ekta Rewar received her B.Tech degree in Electronics and Communication Engineering from Jagannath Gupta Institute of Engineering and Technology, Rajasthan Technical University, India in 2011 and currently is a M. Tech (Signal Processing) student in Mody Institute of Technology and Science (Deemed University), Rajasthan, India. Her research interest lies in Signal Processing \& Image Processing.

S.K.Lenka received his B.E. from Utkal University in 1994, M.Tech. from Kalinga University, $\mathrm{PhD}$ degree from Behrampur University, India. He is currently Professor and Head, Department of Information Technology, MITS University, Lakshmangarh, Rajasthan. His research interest lies in Digital Image Processing, Computer Architecture \& Information Security
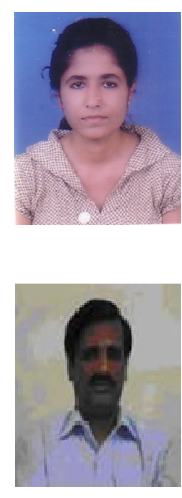